\section{Odor stimuli, training procedures, and performance in a $\mathbf{T}$-maze}

\author{
PHILIP F. SOUTHALL and CHARLES J. LONG \\ Memphis State University, Memphis, Tenn. 38111
}

This study demonstrates that rats can use odor cues to learn a T-maze discrimination task, thus supporting the findings of a previous study. Furthermore, only a very small quantity of dried food $(45 \mathrm{mg})$ was needed to supply odor for the discrimination. The use of odor cues arising from the food reinforcement can occur during initial training in a T-maze and is not dependent on training procedures which first require animals to use strong odor stimuli to make a discrimination. No definite conclusion about the role of animal odors as cues for a discrimination was possible with the data from this study.

Odor stimuli emanating from a food reward were found to serve as effective discriminative stimuli for rats in a T-maze (Southall \& Long, 1969). In this initial study, rats were easily trained to make the correct choice in a $T$-maze where $20 \%$ solutions of either amyl acetate or butyric acid served as discriminative stimuli. Once animals were responding at a significant level of performance, a stepwise reduction in odor intensity was instituted until only the odor of a single $45-\mathrm{mg}$ Noyes food pellet remained as the discriminative stimulus. While these animals could maintain a discrimination at near perfect levels to the odor of a single food pellet, the question remains as to whether very weak odors would be likely to serve as relevant stimuli during initial training in situations where animals were not subjected to a systematic reduction in intensity of the odor serving as the discriminative stimulus.

The present experiment was designed to repeat the first experiment with much lower intensities of odor and, in addition, attempted to answer the following questions: (1) Does the training procedure affect the ability of rats to use odor stimuli? (2) Can rats utilize animal odors as additional discriminative stimuli in learning to make the correct response in a T-maze?

\section{SUBJECTS}

The animals consisted of 12 male Sprague-Dawley rats, approximately 100 days of age at the beginning of the experiment. They were housed individually and maintained on ad lib water. Following 1 day of adaptation to the laboratory environment and 2 weeks prior to the start of the experiment, the animals were placed on a feeding schedule of $15 \mathrm{~g}$ of powdered Purina Lab Chow delivered each day immediately following their scheduled running time.

$$
\text { APPARATUS }
$$

The apparatus used in this experiment consisted of a T-maze, 3.75 in. wide and 6.75 in. high throughout. The lengths of the stem, arm, and goalboxes were 18,2 , and 13 in., respectively. The floor and walls of the maze were made of black Plexiglas, and each section of the maze was covered with a hinged transparent Plexiglas lid. Opaque Plexiglas sliding doors, with $0.5 \times 2.5$ in. cutouts at both the top and bottom to allow air passage, were placed across the runways at the startbox and goalboxes. Retractable Plexiglas foodcups were accessible through a hole cut in the base of a recess located at the distal end of each goalbox. The end of each arm of the maze was fitted with a plenum where background air and the odor stimulus were mixed.

An electric fan was used to pull filtered background air simultaneously into each goalbox and out of the maze through the grid floor of the startbox.

Food odor was obtained from the food reward located in the goalbox. Amyl-acetate-odorized air was produced by bubbling odor-free air through a bottle containing a $0.5 \%$, $0.1 \%$, or $0.05 \%$ solution of amyl acetate in a relatively odorless solvent (di-ethyl phtalate). The apparatus for injecting the odorized air into the plenum has been described in detail elsewhere (Long \& Tapp, 1968). All solutions of amyl acetate were brought to a temperature of $20^{\circ} \mathrm{C}$ before starting the trials.

An odor gradient was established between each goalbox and the startbox by injecting odorized air at a rate of $50 \mathrm{ml} / \mathrm{min}$ into a much larger volume of background air which flowed from the filter into each goalbox at a volume ranging between 17.4 and 21.5 liters $/ \mathrm{min}$ and a velocity ranging from 42 to $52 \mathrm{ft} / \mathrm{min}$. The odorized air was diluted by a factor varying from $2.33 \times 10^{-3}$ to $2.87 \times 10^{-3}$ as it mixed with thebackground air. Each day, just prior to the first trial, the background airflow was checked with a Model AM-12 Hastings air meter, and the injection rate of odorant was checked with a Manostat No.36-541-22 flow meter. The apparatus was cleaned once each day with TSP immediately after all trials had been administered to all animals. PROCEDURE

Each animal was trained to discriminate the correct goalbox by utilizing an odor gradient as a discriminative stimulus to obtain a reward consisting of four $45-\mathrm{mg}$ Noyes food pellets. For Groups 1 and 2, amyl-acetate-odorized air $(0.5 \%$ concentration) was present in conjunction with food odor as the discriminative stimulus, while for Group 3, only the odor pellets served as the discriminative stimulus. The animals were paired randomly and assigned to one of three groups. In order to study the possibility that animal odors served as discriminative stimuli, the position of the reward was arranged according to a double-alternation sequence for all animals in one group and randomly (Fellows, 1967) for the other. Animals

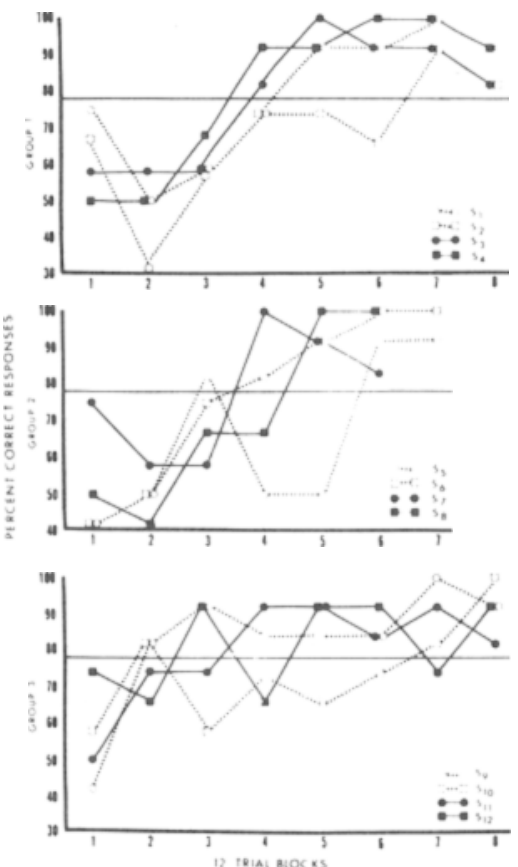

Fig. 1. Performance of individual animals during training expressed as percent correct responses per 12-trial block. Group 1 animals were trained (Blocks 1-8) with amyl acetate (0.5\%) plus food odor. Group 2 animals were trained the same as Group 1. Group 3 animals were trained with food odor only. Rewards were presented in random (open symbols) and double alternation (closed symbols) sequences. 

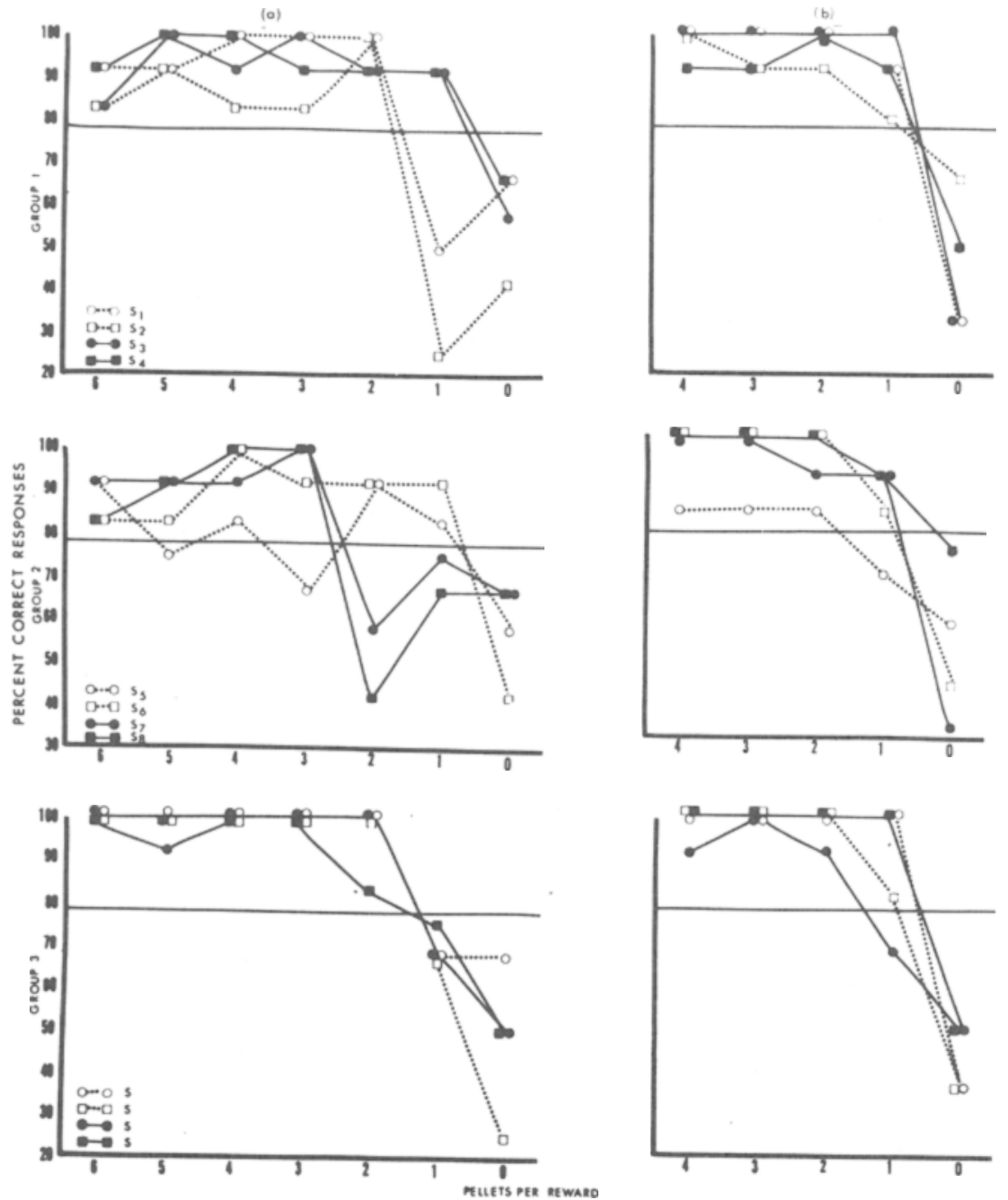

Fig. 2. Percent correct responses made during pellet reduction series. Pellets were reduced by one on each succeeding block of trials, beginning with six pellets (a) and ending with zero pellets for Group 1, Group 1, and Group 3. The series was repeated $(b)$ for the respective groups, beginning with four pellets per reward. Rewards were presented in random (open symbols) and double alternation (closed symbols) sequences.

were run in pairs so that two animals from any given group received the same position sequence. Each pair received daily blocks of 12 trials before another pair recieved its trials; animals in pairs received trials alternately. Training was continued until both animals in a pair had attained a criterion of 10 or more correct responses in 12 trials for at least 2 consecutive days.

\section{Training Phase}

Animals were given one pretraining trial on the first training day, where they were allowed to explore the maze and consume the rewards from both baited cups.

On subsequent trials, the animals were retained in the startbox for $12 \mathrm{sec}$ and in the goalbox for $15 \mathrm{sec}$ and then returned to a transfer cage. the conditions in Group 3 remained the same as during training, with only food odor serving as the discriminative stimulus.

A final test situation was designed to assess the animals' olfactory sensitivity to food pellets. To do this, the number of food pellets available which served as reward and provided odors which could serve as discriminative stimuli were reduced by one pellet for each successive block of 12 trials. On the final test day, the pellets were not present in the $T$-maze but were presented after the animal made the correct choice.

RESULTS

The percent correct results during training for blocks of 12 trials for individual animals in the three groups are depicted in Fig. 1. As can be seen from this figure, all 12 of the animals rapidly acquired the correct response at a significant level of performance (above $78 \%$ correct choice). This $78 \%$ criterion for determining significance represents the .05 confidence limit for a binomial distribution with 12 categories. During early training trials, the performance of the animals in Group 3, trained only with food odor as the discriminative stimulus, tended to approach the required level of significance sooner than the other two groups.

When the amyl acetate was removed from the stimulus complex, leaving only food odor, all animals continued to perform at a significant level. This was true both when the transition was effected suddenly, as with Group 1, or decrementally, as with Group 2.

The performance of individual animals during sensitivity determinations and during a replication of the sensitivity determinations are shown in Fig. 2. As can be seen in this figure, the performance for all groups was significantly different from chance, and the performance of all animals in all groups improved on replication. In the initial reduction (Fig. 2), 10 or 12 animals were able to maintain a high degree of discrimination, with only two food pellets providing the odor cues. On replication (Fig. 2), all but two of the animals performed at a level significantly different from chance with only one pellet present. In both of the reduction series, all animals' performances dropped to a chance level when no food odor was presented, indicating that they were using odor cues to make the discrimination. In the initial reduction series, animals in Group 3, trained only with food odor, exhibited less variation in performance than did the animals in Groups 1 and 2. However, when the reduction series was repeated, there was virtually no 
observable difference between the two training conditions (Fig. 2).

The order of errors between the two training procedures was examined to study the possible role of animal odor cues as discriminative stimuli. However, examination of the patterns of errors did not suggest any significant following behavior. This was due primarily to the fact that the animals rapidly acquired the discrimination; thus, there were too few errors to permit a meaningful analysis. The animals did apparently show signs of learning the double alternation series $\left(x^{2}=5.91 ; p<.05\right)$. DISCUSSION

Are Odor Stimuli Important in Maze Learning?

The rapid acquisition of a significant level of performance to odor stimuli found in this study demonstrates, contrary to most earlier findings, that rats can easily learn to make the correct discrimination in a T-maze to odor cues. Furthermore, it supports the earlier finding that the odor from only a small (45 mg) quantity of dried food may serve as an effective discriminative stimulus (Southall \& Long, 1969). This finding also supports Miller \& Erickson's (1966) conclusion that it is very important to introduce controls for odors, since they may serve as significant cues in situations of which we are not aware. When other more preferred sensory modes are functionally blocked or controlled, the olfactory system may be reliably used to make discriminations which involve odorous substances.

Can Rats Utilize Animal Odors in

Learning to Make a Discrimination in a T-maze?

Analysis of the patterning of errors for pairs of animals during training and during the two pellet-reduction series did not reveal clear evidence that animal odors were serving as discriminative cues. The use of animal odors could have been obscured by any of several factors. The task was such that odors could have been used either positively, negatively, or both positively and negatively by the animal on a trial to indicate whether or not the animal of the preceding trial received a reward in the goalbox which it entered. Furthermore, the rapidity with which the animals learned the discrimination in the $T$-maze resulted in too few errors for successful analysis of the patterning of errors. Finally, the stimulus odor cues that were presented may have been more important than animal odors, with the result that the animals did not need to rely on animal odors as discriminative cues.

This study, then, does not negate the possibility that animal odors are used as discriminative stimuli, as Ludvigson \& Sytsma (1967) have suggested.

Does the Training Procedure

Affect the Ability of Rats to Use Odor Stimuli?

Earlier research (Southall \& Long, 1969 ) indicated that animals could rapidly learn a discrimination to strong odor, and the same results might not have occurred if a weaker odor had been used in the initial training. It was a surprising finding that amyl acetate did not improve the performance over the odor from the food pellets. In fact, the results suggest that some animals can perform better with only food odor present on the early trials, possibly because the food odor had previously been established as the conditioned reinforcing stimulus. The presence of amyl acetate blocked the food odor, and conditioned reinforcing properties for this "novel" stimulus had to be acquired before the animal responded to it significantly in order to obtain the food reward.

An examination of Fig. 1 reveals that, although the performance of all animals is relatively homogeneous, the most erratic performance in most instances comes from those animals whose rewards were randomly presented. However, the consistency of performance for all animals increased with training. This improvement in consistency also occurred with a repetition of the pellet-reduction series (Fig. 2). Not only were the animals able to discriminate significantly with fewer pellets, but they maintained a higher and more consistent level of performance before a sudden failure in discriminative ability .

\section{REFERENCES}

FELLOWS, B. J. Chance stimulus sequences for discrimination tasks. Psychological Bulletin, 1967,67, 87-92.

LONG, C. J.. \& TAPP, J. T. An apparatus for the assessment of the reinforcing properties of odors in small animals. Journal of the Experimental Analysis of Behavior, 1968, 11, 49-51.

LUDVIGSON, H. W., \& SYTSMA, D. The sweet smell of success: Apparent double alternation in the rat. Psychonomic Science, 1967, 9, 283-284.

MILLER. S. D.. \& ERICKSON, R. P. The odor of taste solutions. Physiology \& Behavior, 1966, 1, 1945-1946.

SOUTHALL, P. F., \&ONG, C. J. Odor cues in a maze discrimination. Psychonomic Science, 1969, 16, 126-127. 